\title{
Computed tomography-guided cutting needle pleural biopsy: Accuracy and complications
}

\author{
YI-YUAN CAO, NA FAN, FEN XING, LI-YING XU, YAN-JUAN QU and MEI-YAN LIAO \\ Department of Radiology, Zhongnan Hospital of Wuhan University, Wuhan, Hubei 430071, P.R. China
}

Received March 25, 2014; Accepted October 16, 2014

DOI: $10.3892 /$ etm.2014.2078

\begin{abstract}
In cases of pleural lesion, tissue samples can be obtained through thoracoscopy or closed needle biopsy for histopathological analysis. Cutting needle biopsy is a relatively recent addition to these techniques. The aim of this study was to evaluate the diagnostic accuracy and safety of computed tomography-guided cutting needle pleural biopsy (CT-CNPB), as well as the associated complications, in patients with pleural lesion. This study was a retrospective analysis of 92 percutaneous CT-CNPBs on 90 patients between March 2008 and May 2013. For group comparisons, $\chi^{2}$ tests were used to detect the risk factors for diagnostic accuracy (false-negative rate). Of the 92 CT-CNPBs, malignant lesions were diagnosed in 55 cases (mesothelioma in 12 , metastatic pleural disease in 36 , synoviosarcoma in one, indeterminate-origin disease in one and false-negative lesion in five) and benign pleural disease was diagnosed in 37 cases (inflammation in 15, tuberculosis in 10, granuloma in three, solitary fibrous tumor in two, hematoma in one, fungus in one and indeterminate-origin disease in five). The sensitivity of diagnostic malignant lesion was $90.9 \%$, and the specificity and positive and negative predictive values were 100,100 and $88.1 \%$, respectively. The overall diagnostic accuracy was $94.6 \%$. A specific diagnosis was achieved in $89.1 \%$ of malignant lesions and $86.4 \%$ of benign lesions. Univariate analysis of the risk factors affecting accuracy (false-negative rate) did not reveal any significant differences (all $\mathrm{P}>0.05$ ). The complication rates were $6.5 \%$ for pneumothorax, $8.7 \%$ for hemorrhage and $1.1 \%$ for hemothorax. In conclusion, CT-CNPB is a safe and accurate diagnostic technique that can be recommended as the primary method of diagnosis in patients with pleural thickening or lesions observed by CT scan.
\end{abstract}

Correspondence to: Dr Mei-Yan Liao, Department of Radiology, Zhongnan Hospital of Wuhan University, 169 Donghu Road, Wuhan, Hubei 430071, P.R. China

E-mail: meiyanliaocn@163.com

Key words: pleural biopsy, pleural disease, cutting needle, computed tomography-guided

\section{Introduction}

The diagnostic approach to pleural disease is a relatively neglected aspect of modern thoracic medicine, despite the fact that pleural disease affects $\sim 300$ subjects per 100,000 individuals per year worldwide $(1,2)$. If a clinical suspicion of malignancy is high in patients with pleural effusion, cytological examination of pleural fluid samples is recommended; the diagnostic yield for malignancy with pleural cytology is 50-60\%, which falls to $30 \%$ in effusions associated with malignant mesothelioma (MM) (3). Tumor type and the availability of reliable immunocytochemistry may influence the yield; for example, the cytological detection rate for adenocarcinoma is higher than that for squamous cell carcinoma, mesothelioma or lymphoma (4). When the distinction between primary and metastatic tumors is addressed, morphological criteria alone are not sufficient for a definite diagnosis of MM (5). In instances when cytology is non-diagnostic $(3,4)$, closed percutaneous needle biopsy has traditionally been performed blindly using a reverse-beveled needle, such as an Abrams or Ramel needle; however, blind closed pleural biopsy has a relatively modest diagnostic yield of $<60 \%$ for pleural malignancy (6).

Medical thoracoscopy enables the direct examination of the pleura and biopsies taken under direct vision and has a diagnostic yield superior to that of blind closed pleural biopsy and thoracocentesis. The diagnostic yield is $91-95 \%$ for malignant disease and can reach $100 \%$ for pleural tuberculosis. Furthermore, although medical thoracoscopy is more invasive and expensive, complications occur only infrequently $(4,7)$. Despite this, the most efficient and cost-effective approach to pleural lesions remains unclear and controversial, particularly in cases requiring the acquisition of pleural tissue. Recent studies have proposed that image guidance may significantly increase the diagnostic yield while simultaneously decreasing the risk of complications. It has also been suggested that real-time computed tomography-guided cutting needle pleural biopsy (CT-CNPB), performed by a radiologist, is a promising technique for sampling the pleura, as it can improve diagnostic sensitivity to $\sim 80 \%$ for pleural malignancy $(8,9,10)$. Image-assisted biopsy is more likely to be diagnostic in the presence of pleural thickening $>10 \mathrm{~mm}$, pleural nodularity, pleural-based mass lesions of $>20 \mathrm{~cm}$ and solid pleural tumors (7,11-16). In the present study, the diagnostic accuracy and safety of CT-CNPB was evaluated in patients requiring pleural tissue sampling. 


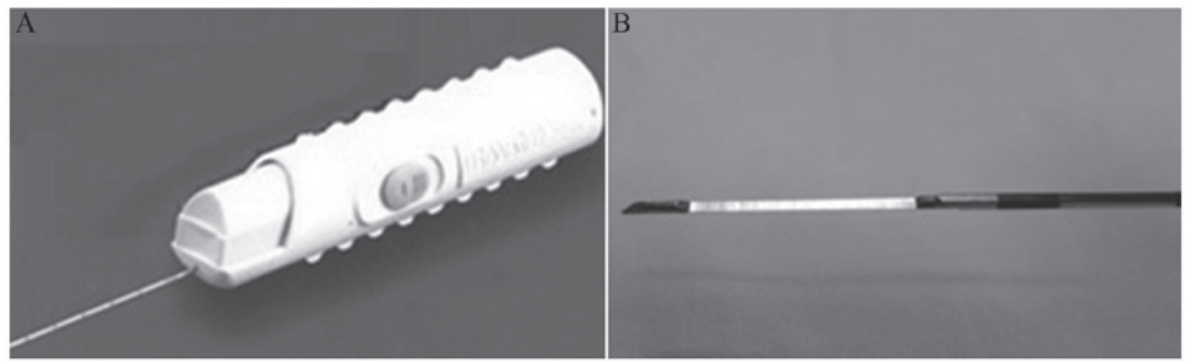

Figure 1. (A) Bard ${ }^{\circledR}$ Max-Core ${ }^{\circledR} 18 \mathrm{G}$ biopsy needle; (B) top slide locked back and biopsy sample notch exposed.

\section{Materials and methods}

Study population. This study was a retrospective analysis of 92 percutaneous CT-CNPBs on 90 patients between March 2008 and May 2013. All procedures were performed by the same two radiologists who were experienced in performing CT-guided pleural biopsies. Percutaneous CT-CNPB was indicated in any patient with a plural lesion requiring biopsy. Patients excluded from the study included patients with lung-based tumors or no final diagnosis. Each patient provided their written informed consent prior to the procedure. This study was conducted in accordance with the Declaration of Helsinki and with approval from the Ethics Committee of Zhongnan Hospital of Wuhan University (Wuhan, China).

Procedure. A CT scan of the chest (Somatom Sensation16; Siemens Healthcare, Forchheim, Germany) was initially performed to identify the lesion. The patient was then positioned in the supine, prone or lateral position to minimize puncture depth. An initial localization scan with a low-dose technique (Lung CARE series: 20-50 mA; $120 \mathrm{kV}$; scanning field, 30-60 mm; Siemens Healthcare) through the region of interest was performed at a slice thickness of $5 \mathrm{~mm}$ and viewed on both lung and soft-tissue windows. Localization was performed subsequent to the review of the CT images using laser positioning and skin markers (Biopsy single series: $50 \mathrm{~mA} ; 120 \mathrm{kV}$; thickness, $10 \mathrm{~mm}$; scanning field, $10 \mathrm{~mm}$; Siemens Healthcare) to indicate the site of needle entry and the direction of approach for the biopsy. Subsequent to ensuring that the direction of needle approach was perpendicular to the chest wall, the thickness of the thoracic wall was measured from the skin marker to the pleural surface to determine the depth of anesthesia to be administered and the depth of needle insertion. Using an aseptic technique, local anesthetic (lignocaine 1\%) was administered. An 18-gauge coaxial automated cutting needle (Bard ${ }^{\circledR}$ Max-Core ${ }^{\circledR}$ biopsy needle; C.R. Bard, Inc., Tempe, AZ, USA) (Fig. 1) was then introduced into the soft tissues without traversing the pleural surface (Figs. 2 and 3). The position of the pleural lesion in relation to the tip of the needle and the precise distance to the margin of the lesion (Figs. 2 and 3) were optimized using sequential CT scanning (Biopsy single series: $50 \mathrm{~mA} ; 120 \mathrm{kV}$; thickness, $4.5 \mathrm{~mm}$; scanning field, $13.5 \mathrm{~mm}$; Siemens Healthcare). According to the precise information obtained from the repeat CT scan, the trajectory of the needle was adjusted, and the biopsy gun was directly advanced into the lesion and fired to obtain a core of tissue. The procedure was stopped immediately if the patient complained of discomfort,

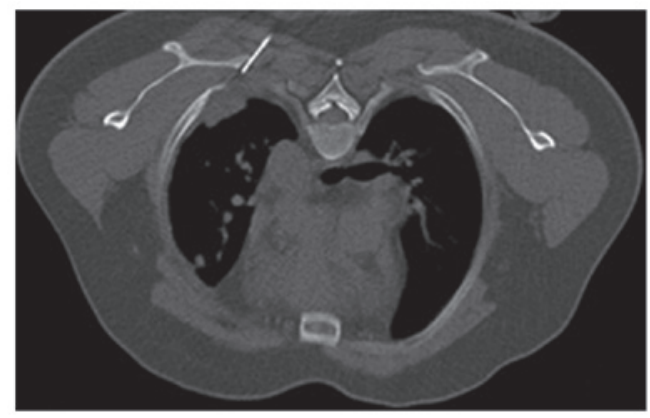

Figure 2. Male, aged 39 years, the first result of the computed tomography-guided cutting needle pleural biopsy was a false-negative lesion; the secondary biopsy led to a diagnosis of metastatic adenosquamous carcinoma.

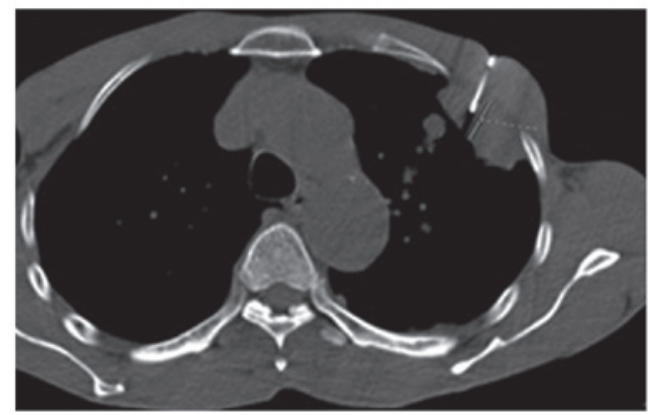

Figure 3. Male, aged 75 years, the result of the computed tomography-guided cutting needle pleural biopsy was malignant mesothelioma.

such as dyspnea, severe cough or hemoptysis. The surgeon then assessed the adequacy of the sample prior to deciding whether to proceed with additional passes. The obtained specimens were placed in formalin solution using a saline-filled syringe. Once the surgeon was satisfied with the samples obtained, immediate post-biopsy CT (Biopsy single series: $50 \mathrm{~mA} ; 120 \mathrm{kV}$; thickness, $4.5 \mathrm{~mm}$; scanning field, $13.5 \mathrm{~mm}$ ) was performed over the region of the biopsy to check for pneumothorax or hemorrhage.

Classification of diagnoses and complications. The biopsy specimens were evaluated by the same experienced pathologist. The cases were categorized primarily as benign and malignant, and those that were malignant were also categorized according to the cell properties. Immunohistochemical stains were used to differentiate tumors when required. The immunohistochemical markers used were: cytokeratin 7, thyroid transcription factor-1, vimentin, mesothelial cell and calretinin. A positive expression of calretinin, Vim and 
mesothelial cell would indicate that pleural mesothelioma occured, whereas, positive expression of cytokeratin 7 and thyroid transcription factor-1 would support that lung metastasis has occured. The pathologist also stained the specimens with Ziehl-Neelsen (Baso Diagnostics Inc., Zhuhai, China) to investigate for acid-resistant bacilli. A specific histological diagnosis was defined as a definite histological type; the results were considered non-specific when no particular diagnosis could be established from the specimen obtained and in false-negative cases of malignancy. The final diagnosis was confirmed at surgery. Histological findings obtained by biopsy were compatible with the patient's clinical manifestations of disease.

The presence of pneumothorax was assessed by a low-dose $\mathrm{CT}$ technique. If the patient was clinically stable, he or she was kept under medical observation for $12 \mathrm{~h}$. All patients had a chest radiograph performed $4 \mathrm{~h}$ after the procedure or sooner if they became symptomatic. Pneumothorax (17) and hemorrhage (18) were graded as mild, moderate or severe.

Statistical analysis. Details regarding the nature of the lesion (lesion location and thickness), the procedure itself (false-negative rate, requirement for CT contrast enhancement to distinguish a lesion from the thorax wall and vessels and number of attempts) and complications were calculated. Categorical variables are presented as counts and percentages, and $\chi^{2}$ tests were conducted for group comparisons. Logistic regression models were performed to detect the risk factors for diagnostic accuracy (false-negative rate). Factors with a significance level of $\mathrm{P}<0.05$ in the univariate analyses were included in the multivariate model. A two-sided P-value of $<0.05$ was considered statistically significant. Statistical Analysis System (SAS) version 9.2 statistical software (SAS Inc., Cary, NC, USA) was used for all analyses.

\section{Results}

Patient characteristics. In the present study, 90 patients, including 53 males and 37 females (mean age, $55.2 \pm 14.5$ years) underwent 92 CT-CNPBs. There were 64 (71.1\%) inpatients and 26 (28.9\%) outpatients. Pleural thickening in the 92 cases varied between 6 and $99 \mathrm{~mm}$, and 23 cases (25\%) had pleural effusion. A total of 21 cases underwent $\mathrm{CT}$ contrast enhancement. The number of attempts ranged between two and four, and the duration of the procedure was $16 \pm 3 \mathrm{~min}$.

Biopsy yield. The distribution of diagnoses for cases included in the study is shown in Table I. Immunohistochemical stains were used in 19 cases. The sensitivity of diagnostic malignant lesion was $90.9 \%$ (50/55), the specificity was $100 \%$ (37/37), the positive predictive value was $100 \%(50 / 50)$ and the negative predictive value was $88.1 \%$ (37/42). The overall diagnostic accuracy was $94.6 \%$ (87/92). A specific histological diagnosis was achieved in $89.1 \%$ (49/55) of malignant lesions and $86.5 \%$ (32/37) of benign lesions. The non-specific results and confirmation methods are shown in Table II.

There were five false-negative cases in 55 malignant cases. Univariate analysis of the risk factors affecting accuracy (false-negative rate) did not reveal any significant differences (all P>0.05) (Table III).
Table I. Distribution of the diagnoses of the 92 cases included in the study.

A, Malignant, $\mathrm{n}=55(59.8 \%)$

\begin{tabular}{lc}
\hline Diagnosis & $\mathrm{N}(\%)$ \\
\hline Mesothelioma & $12(13.0)$ \\
Metastatic pleural disease & $36(39.1)$ \\
Adenocarcinoma & $24(26.1)$ \\
Squamous carcinoma & $5(5.4)$ \\
Clear cell carcinoma & $2(2.2)$ \\
Adenosquamous carcinoma & $1(1.1)$ \\
Small cell carcinoma & $1(1.1)$ \\
Carcinocarcoma & $1(1.1)$ \\
Plasmocytoma & $1(1.1)$ \\
Osteosarcoma & $1(1.1)$ \\
Synovial sarcoma & $1(1.1)$ \\
Malignant cell & $1(1.1)$ \\
False-negative lesion & $5(5.4)$ \\
\hline
\end{tabular}

B, Benign, $n=37(40.2 \%)$

\begin{tabular}{lc}
\hline Diagnosis & $\mathrm{N}(\%)$ \\
\hline Inflammation & $15(16.3)$ \\
Tuberculosis & $10(10.9)$ \\
Granuloma & $3(3.3)$ \\
Solitary fibrous tumor & $2(2.2)$ \\
Hematoma & $1(1.1)$ \\
Fungus & $1(1.1)$ \\
Indeterminate-origin disease & $5(5.4)$
\end{tabular}

Complications. Pneumothorax occurred in six cases $(6.5 \%)$ in the group with a pleural thickness of $>30 \mathrm{~mm}$; five (5.4\%) of the cases were graded as mild and one case (1.1\%) was graded as severe and required a chest drain. No further treatment was required for these cases. Mild lung hemorrhage around the entry site occurred in eight cases (8.7\%) subsequent to the CT-CNPB. One patient (1.1\%), who had a history of long-term oral aspirin administration, suffered from a hemothorax, which required tube thoracostomy. No cases required blood transfusion and no patients succumbed in the three days following the procedure. No patients required additional analgesics due to the pain.

\section{Discussion}

Pleural diseases are a frequently occurring medical problem and the differential diagnosis is wide. Pleural aspiration is recommended as the first diagnostic procedure in patients with pleural effusion. This procedure is simple and safe and can often be performed at the bedside or in the clinic. The success rate of ultrasound-guided pleural aspirations can be $\leq 97 \%$ (16). For cases in which malignancy is suspected, fluid should be sent for cytological examination. The diagnosis of 
Table II. Non-specific specimen results and the final diagnoses.

\begin{tabular}{|c|c|c|c|}
\hline Case & Specimen results & Confirmation methods & Final diagnoses \\
\hline 1 & Malignant cell & Secondary biopsy & Adenocarcinoma \\
\hline 2 & Atypical mesothelial proliferation & Surgery & Adenosquamous carcinoma \\
\hline 3 & Atypical mesothelial proliferation & Secondary biopsy & Adenocarcinoma \\
\hline 4 & Fibrous tissue & Surgery & Adenosquamous carcinoma \\
\hline 5 & Fibrous tissue & Clinical manifestation: Lesion enlarged & $\begin{array}{l}\text { Metastatic pleural disease } \\
\text { of lung adenocarcinoma }\end{array}$ \\
\hline 6 & Fibrous tissue & Adenocarcinoma cell in hydrothorax & $\begin{array}{l}\text { Metastatic pleural disease } \\
\text { of ovarian adenocarcinoma }\end{array}$ \\
\hline $7 / 8$ & $\begin{array}{l}\text { Glassy degeneration tissue and } \\
\text { fibrous connective tissue }\end{array}$ & Clinical follow-up in two years & Benign lesion \\
\hline 9 & Necrotic tissue & Absorbed after antituberculosis therapy & Tuberculosis \\
\hline 10 & Fibrous connective tissue & Surgery & Inflammation \\
\hline 11 & Fibrous tissue hyperplasia & Surgery & Solitary fibrous tumor \\
\hline
\end{tabular}

Table III. Univariate analysis of the risk factors affecting accuracy (false-negative rate).

\begin{tabular}{|c|c|c|}
\hline & $\begin{array}{l}\text { False-negative } \\
\text { cases/total }(\%)\end{array}$ & $\mathrm{P}$-value \\
\hline Gender & & 1.000 \\
\hline Male & 3/31 (9.67) & \\
\hline Female & $2 / 24(8.33)$ & \\
\hline Age, years & & 0.878 \\
\hline$\leq 50$ & $1 / 14(7.14)$ & \\
\hline $51-60$ & $1 / 16(6.25)$ & \\
\hline $61-70$ & $2 / 14(8.33)$ & \\
\hline$>70$ & $1 / 11(9.09)$ & \\
\hline Lesion location & & 0.061 \\
\hline Right upper & $0 / 10(0.00)$ & \\
\hline Right middle & $0 / 2(0.00)$ & \\
\hline Right lower & $1 / 18(5.56)$ & \\
\hline Left upper & $0 / 11(0.00)$ & \\
\hline Left lower & $4 / 14(28.57)$ & \\
\hline Lesion thickness, mm & & 0.144 \\
\hline$\leq 10$ & 2/6 (33.33) & \\
\hline $11-20$ & $0 / 21(0.00)$ & \\
\hline $21-30$ & $1 / 6(16.67)$ & \\
\hline $31-40$ & $1 / 9(11.11)$ & \\
\hline$>40$ & 1/13 (7.69) & \\
\hline Number of attempts & & 0.747 \\
\hline$\leq 2$ & 1/9 (11.11) & \\
\hline 3 & $3 / 40(7.5)$ & \\
\hline$\geq 4$ & 1/6 (16.67) & \\
\hline Pleural effusion & & 0.649 \\
\hline Yes & $1 / 19(5.26)$ & \\
\hline No & 4/36 (11.11) & \\
\hline Contrast enhancement & & 0.592 \\
\hline Yes & 2/14 (14.29) & \\
\hline No & $3 / 41(7.31)$ & \\
\hline
\end{tabular}

MM, metastasis or benign mesothelial proliferation in effusion samples is often challenging for medical professionals due to sampling problems (few malignant cells shedding and hemorrhagic or inflammatory effusion) and/or errors in interpretation. The reported sensitivity for the cytological diagnosis of MM ranges between 31.9 and $86.3 \%$ for malignancy without further specification and between 11.7 and $75.3 \%$ for a correct diagnosis of primary neoplasm (20). A significant proportion of biopsies may be technically inadequate and there is a significant false-negative rate of $35-50 \%$ (18). Furthermore, a percentage of false-positive cases still occur, with reactive mesothelial cells mimicking malignancy (5). In the present series, $25 \%$ of cases had pleural effusion.

The diagnostic yield of unaided (blind) closed pleural biopsy for pleural malignancy is a relatively modest $<60 \%$. Of note is the fact that the overall diagnostic yield for malignancy is only increased by $7-27 \%$ when compared with pleural fluid cytology (22). CNPB is a relative recent technique to be adopted. Image-guided cutting needle biopsy of mass lesions associated with pleural effusion is a well-validated modality, producing diagnostic yields higher than those for closed pleural biopsy. A contrast-enhanced thoracic CT scan of a patient with a pleural effusion may show focal areas of abnormal thickening (7). A study by Maskell et al (12) found that CT guidance significantly increased the diagnostic yield with regard to pleural thickening. In their study, CT-CNPB had a sensitivity of $87 \%$, whereas unaided Abrams needle biopsy had a sensitivity of $44 \%$ $(\mathrm{P}=0.02)$ (12). Furthermore, Adams and Gleeson (24) previously found that CT-guided biopsies had a sensitivity of $93 \%$ for MM. The sensitivity of diagnostic malignant lesion calculated in the present study is among the highest of those previously published, and there were no false-positive cases in the group.

For pleural thickening of $\leq 5 \mathrm{~mm}$, the sensitivity of CT-guided needle biopsy is $75 \%$. The frequency of non-diagnostic biopsies ranges between 0 and $9 \%(11,25)$; therefore, if a CT-guided biopsy is performed in cases with minor pleural thickness, there may be a lower probability that a sufficient amount of tissue will be obtained. The rate of non-diagnostic pleural biopsies in the present series was $0 \%$, and the rate of non-specific histological 
diagnosis was $12.0 \%$ (11/92). The non-specific cases may have been a result of inadequate biopsy samples and lesion complexity. In certain cases, biopsy would be insufficient for the final diagnosis; these cases would require further clinical and radiological investigations, as well as follow-up.

CT-guided biopsy may significantly increase the diagnostic yield while decreasing the risk of complications; the procedure can be performed in outpatient conditions and can be used for patients without pleural effusion. Furthermore, CT-CNPB can be performed in patients with pleural thickening in the absence of pleural fluid, which is not convenient when using an Abrams needle. The main complication associated with the procedure is pneumothorax. Although pneumothorax occurs in $\leq 15 \%$ of patients undergoing biopsies, very few require intervention. Other complications may include site pain (1-15\%), vasovagal reaction with potential syncope $(1-5 \%)$, hemothorax $(<2 \%)$ and site hemorrhage with hematoma formation $(<1 \%)(4)$. In the present series, the pneumothorax rate was $6.5 \%$, and one patient required a chest drain. Bleeding occurred in $8.7 \%$ of the cases at the time of biopsy, although no subsequent blood transfusions were necessary. When an $17 / 18$ or $20 / 21 \mathrm{G}$ needle with a $2-\mathrm{cm}$ throw is utilized to sample minimal pleural thickening in the absence of a pleural effusion, the visceral pleura and adjacent lung are likely to be traversed. A number of the observed pneumothoraces may have been a result of the introduction of air by the biopsy or drain rather than due to a direct communication with the airway.

Thoracoscopy has the advantage that it enables direct visualization of the pleura, although the visceral and parietal pleura must not be adherent for the technique to be performed. In a previous study, the CT-guided pleural Abrams needle biopsy group had a diagnostic sensitivity of $87.5 \%$, whereas the medical thoracoscopy group had a sensitivity of $94.1 \%$ $(\mathrm{P}=0.252)$. Furthermore, $\mathrm{CT}$-guided Abrams needle biopsy had a sensitivity of $95 \%$ in cases with pleural thickening $\geq 1 \mathrm{~cm}$, which was similar to the sensitivity obtained with thoracoscopy (96\%). Thoracoscopy achieved higher sensitivity in cases with $<1 \mathrm{~cm}$ thickening ( 93 vs. $82 \%, \mathrm{P}=0.42$ ). The authors concluded that CT-guided Abrams needle biopsy should be used as the primary method of diagnosis in patients with pleural thickening or lesions observed by CT scan, but suggested that patients with the appearance of only pleural fluid on the CT scan may still benefit from primary medical thoracoscopy (14). Medical thoracoscopy has the advantage that it may additionally be used for therapeutic purposes, for example for the direct insufflation of talc in order to achieve pleurodesis and the breakdown of loculations. Surgical thoracoscopy, requiring complete deflation of a lung and superior access for therapeutic interventions, is significantly more invasive and expensive (4).

In conclusion, CT-CNPB is a safe and accurate diagnostic technique. It is suggested that the present method of CT-CNPB be used as a first diagnostic evaluation in those cases with pleural thickness or pleural lesion observed in the thoracic CT scan.

\section{Acknowledgements}

This study was supported by the key foundation of Hubei Nature Scientific Funds (grant no. 2012FFB04414).

\section{References}

1. Du Rand I and Maskell N: Introduction and methods: British Thoracic Society Pleural Disease Guideline 2010. Thorax 65 Suppl 2: ii1-ii3, 2010.

2. Koegelenberg CF and Diacon AH: Pleural controversy: closed needle pleural biopsy or thoracoscopy-which first? Respirology 16: 738-746, 2011.

3. Renshaw AA, Dean BR, Antman KH, Sugarbaker DJ and Cibas ES: The role of cytologic evaluation of pleural fluid in the diagnosis of malignant mesothelioma. Chest 111: 106-109, 1997.

4. Hooper C, Lee YC and Maskell N; BTS Pleural Guideline Group: Investigation of a unilateral pleural effusion in adults: British Thoracic Society pleural disease guideline 2010. Thorax 65 Suppl 2: ii4-ii17, 2010.

5. Fassina A, Fedeli U, Corradin M, Da Frè M and Fabbris L: Accuracy and reproducibility of pleural effusion cytology. Leg Med (Tokyo) 10: 20-25, 2008.

6. Tomlinson JR: Invasive procedures in the diagnosis of pleural disease. Semin Respir Med 9: 30-60, 1987.

7. Lee P, Hsu A, Lo C and Colt HG: Prospective evaluation of flex-rigid pleuroscopy for indeterminate pleural effusion: Accuracy, safety and outcome. Respirology 12: 881-886, 2007.

8. Sconfienza LM, Mauri G, Grossi F, et al: Pleural and peripheral lung lesions: comparison of US- and CT-guided biopsy. Radiology 266: 930-935, 2013.

9. Chira R, Chira A and Mircea PA: Intrathoracic tumors in contact with the chest wall - ultrasonographic and computed tomography comparative evaluation. Med Ultrason 14: 115-119, 2012.

10. Ferretti GR, Busser B, de Fraipont F, Reymond E, et al: Adequacy of CT-guided biopsies with histomolecular subtyping of pulmonary adenocarcinomas: influence of ATS/ERS/IASLC guidelines. Lung Cancer 82: 69-75, 2013

11. Gupta S and Madoff DC: Image-guided percutaneous needle biopsy in cancer diagnosis and staging. Tech Vasc Interv Radiol 10: 88-101, 2007.

12. Maskell NA, Gleeson FV and Davies RJ: Standard pleural biopsy versus CT-guided cutting-needle biopsy for diagnosis of malignant disease in pleural effusions: a randomised controlled trial. Lancet 361: 1326-1330, 2003.

13. Benamore RE, Scott K, Richards CJ, et al: Image-guided pleural biopsy: diagnostic yield and complications. Clin Radiol 61: 700-705, 2006.

14. Metintas M, Ak G, Dundar E, et al: Medical thoracoscopy vs. CT scan-guided Abrams pleural needle biopsy for diagnosis of patients with pleural effusions: a randomized, controlled trial. Chest 137: 1362-1368, 2010

15. Cheung YC, Chang JW, Hsieh JJ, et al: Adequacy and complications of computed tomography-guided core needle biopsy on non-small cell lung cancers for epidermal growth factors receptor mutations demonstration: 18-gauge or 20 -gauge biopsy needle. Lung Cancer 67: 166-169, 2010.

16. Solomon SB, Zakowski MF, Pao W, et al: Core needle lung biopsy specimens: adequacy for EGFR and KRAS mutational analysis. AJR Am J Roentgenol 194: 266-269, 2010.

17. Yeow KM, See LC, Lui KW, et al: Risk factors for pneumothorax and bleeding after CT-guided percutaneous coaxial cutting needle biopsy of lung lesions. J Vasc Interv Radiol 12: 1305-1312, 2001.

18. Yeow KM, Su IH, Pan KT, et al: Risk factors of pneumothorax and bleeding: multivariate analysis of $660 \mathrm{CT}$-guided coaxial cutting needle lung biopsies. Chest 126: 748-754, 2004.

19. Yang PC, Kuo SH and Luh KT: Ultrasonography and ultrasoundguided needle biopsy of chest diseases: indications, techniques, diagnostic yields and complications. J Med Ultrasound 1: 53-63, 1993.

20. Di Bonito L, Falconieri G, Colautti I, et al: Cytopathology of malignant mesothelioma: a study of its patterns and histological bases. Diagn Cytopathol 9: 25-31, 1993.

21. Poe RH, Israel RH, et al: Sensitivity, specificity and predictive values of closed pleural biopsy. Arch Int Med 144: 325-328, 1984.

22. Nance KV, Shermer RW and Askin FB: Diagnostic efficacy of pleural biopsy as compared with that of pleural fluid examination. Mod Pathol 4: 320-324, 1991.

23. Adams RF and Gleeson FV: Percutaneous image-guided cutting needle biopsy of the pleura in the diagnosis of malignant mesothelioma. Chest 120: 1798-1802, 2001.

24. Adams RF and Gleeson FV: Percutaneous image-guided cutting needle biopsy of the pleura in the presence of a suspected malignant effusion. Radiology 219: 510-514, 2001. 